\title{
Implementación de cartas de control en el paquete estadístico $R$ para el monitoreo de procesos en media con datos autocorrelacionados
}

\author{
Implementation of Control Charts in the Statistical Package $\mathbf{R}$ for \\ Monitoring Processes in Media with Autocorrelated Data
}

Joaquín González国

jgonzalezb@ut.edu.co
Alex Zambrand

azambrano@ut.edu.co

\begin{abstract}
Resumen
Las cartas de control estadístico son gráficos que dentro del control de proceso estadístico permiten monitorear la(s) característica(s) de calidad de un proceso industrial o de servicios y son ampliamente usadas en la actualidad. Para la implementación de las cartas usuales se asume que las observaciones no presentan una estructura de autocorrelación, pero en la práctica esta condición es violada con frecuencia. La presencia de autocorrelación tiene un serio impacto sobre el incremento sustancial en la frecuencia de falsas alarmas en dichas cartas. En los últimos 20 años, varias propuestas de cartas de control se han presentado teniendo en cuenta la estructura de autocorrelación en los datos para su construcción. En este artículo se presenta la construcción y comparación en forma ilustrativa de dos de dichas propuestas, una dada por Alwan \& Roberts (1988) basada sobre los residuales del modelo tipo ARMA que mejor se ajuste a los datos, y la otra dada por Montgomery \& Mastrangelo (1991) basada sobre la estadística EWMA, con el objeto de que sirvan como instrumento de enseñanza y aprendizaje de este tipo de cartas, ya que paquetes especializados en control estadístico de la calidad no tienen implementadas estas cartas. Tal es el caso del paquete estadístico R (R Development Core Team 2009), el cual es usado en este trabajo para la programación e implementación de las cartas mencionadas.
\end{abstract}

Palabras clave: cartas de control estadístico, autocorrelación, modelos ARMA, estadística EWMA, enseñanza y aprendizaje del control de calidad, paquete estadístico R.

\footnotetext{
${ }^{\text {a}}$ Profesor de planta. Universidad del Tolima.

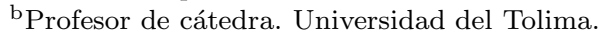




\begin{abstract}
Statistical control charts are graps in statistical process control can monitoring quality characteristic(s) of an industrial process or service and are widely used today. To implement these assumes that the observations do not present an autocorrelation structure, but in practice this condition is violated frequently. The presence of autocorrelation has a serious impact on a substantial operation in the frequency of false alarms. Montgomery \& Mastrangelo (1991) presents the construction of a control chart for autocorrelated data keeping in mind the structure of the EWMA statistic that is a good predictor one step ahead of the observations given. This control chart presents inconveniences when the autocorrelation is negative. In this paper we consider an alternative control chart which takes into account the data model through a time series ARMA family, for the construction of appropriate control limits from the negative correlation structure doesn't cause problems.
\end{abstract}

Key words: Control Charts, EWMA Statistic, Negative Autocorrelated, Time Series, ARMA Models.

\title{
1. Introducción
}

Las cartas de control son una herramienta muy usada dentro del control de procesos estadístico (CPE). Son un instrumento útil para el análisis y monitoreo de la estabilidad de un proceso a través de una(s) característica(s) de calidad, que sirve para detectar patrones anormales en cualquier tiempo. En la práctica se desea controlar el valor promedio de la característica de calidad así como su variabilidad. $\mathrm{El}$ control del valor promedio se hace a través de cartas donde se utilizan medidas de tendencia central, de las cuales la más usual es la media. Cartas tales como $\bar{X}$, CUSUM (sumas acumuladas) (Page 1954) y EWMA (promedios móviles ponderados exponencialmente) (Roberts 1959) son usadas para tal propósito. Las cartas CUSUM y EWMA, son más eficientes en detectar pequeños cambios en la media que la carta $\bar{X}$. Para controlar la variabilidad, frecuentemente se usan medidas como la desviación estándar, como es el caso de la carta S y rangos para la carta R. Estos tipos de cartas de control estadístico son las más usadas en procesos en línea.

La carta EWMA tiene como supuestos que las observaciones provienen de una distribución normal y no están autocorrelacionadas. El segundo supuesto, frecuentemente es violado en la práctica. Esto ocurre cuando las observaciones son tomadas en intervalos cortos de tiempo, muy comunes en procesos industriales químicos. La presencia de autocorrelación tiene un profundo impacto en el funcionamiento de las cartas de control usuales, causando un incremento sustancial en la frecuencia de falsas alarmas.

Montgomery \& Mastrangelo (1991), proponen una carta EWMA que tiene en cuenta la presencia de datos autocorrelacionados en un proceso de control estadístico. 
La carta es construida asumiendo que los datos tienen una estructura de autocorrelación del tipo $\operatorname{ARIMA}(0,1,1)$, para luego aplicarlo a la estadística EWMA, con el fin de obtener límites apropiados donde se tenga en cuenta la autocorrelación de los datos.

Los autores mencionados aseguran que si las observaciones del proceso son modeladas por un miembro de la familia $\operatorname{ARIMA}(p, d, q)$ y además están autocorrelacionadas positivamente y el proceso no cambia tan rápidamente en media, se puede usar la carta para datos autocorrelacionados con una apropiada escogencia de lambda (constante de la estadística EWMA).

Alwan \& Roberts (1988) construyen una carta basada en los residuales del modelo que mejor se ajuste a los datos y los lleva a las cartas tradicionales tipo Shewhart. Jiang et al. (2000) tiene en cuenta la estructura de autocorrelación de los datos en la construcción de los límites de las cartas de control, obteniendo límites fijos.

El presente artículo, está dirigido a presentar en forma ilustrativa y comparativa la construcción de la carta dada por Alwan \& Roberts (1988) además de la carta propuesta por Montgomery \& Mastrangelo (1991), la primera de las cuales no posee inconvenientes ante la presencia de datos autocorrelacionados en forma negativa y se construye llevando las etapas mediante el modelamiento de series de tiempo propuesto por Box \& Jenkins (1976). En contraste, la segunda no tiene en cuenta la estructura tipo ARIMA para la construcción de la carta, en lugar de ello utiliza la estructura de la estadística EWMA.

La ilustración y comparación de las cartas se realizará mediante el uso de códigos y rutinas de programación en el paquete estadístico R ( R Development Core Team 2009).

El artículo está organizado como sigue. En la sección 2 se darán unas consideraciones teóricas haciendo énfasis en la construcción de las cartas de interés. En la sección 3 se da ejemplo mediante simulación de los datos con autocorelación negativa y positiva y se analiza el comportamiento de las dos cartas en cada una de las situaciones dadas. Finalmente, en la última sección se dan las conclusiones y recomendaciones del artículo.

\section{Conceptos teóricos}

Se darán algunos conceptos teóricos que describen en forma general el esquema que deseamos abordar.

\subsection{Cartas de control univariadas en presencia de datos au- tocorrelacionados}

Las primeras cartas de control fueron desarrolladas por Walter A. Shewhart en los años 20 del siglo pasado, basadas sobre la condición de que $f_{\boldsymbol{\theta}}(x)$ es una de las 
pocas distribuciones estándar (normal para datos continuos y binomial o Poisson para datos discretos), donde $\boldsymbol{\theta}$ es el vector de parámetros de la característica de calidad $X$ (por ejemplo, si $X \sim N$ entonces $\boldsymbol{\theta}=(\mu, \sigma)$ ), además que la secuencia de observaciones de $X$ sean independientes. Dichas cartas son aún hoy las más usadas en diferentes ámbitos de aplicación, pero en muchos casos es inapropiada ante la detección ineficiente de pequeños cambios en $\boldsymbol{\theta}$ o ante la violación de algunas de las condiciones dadas para su correcto funcionamiento. El no tener en cuenta el cumplimiento de una o las dos condiciones puede llevar a un serio impacto en el funcionamiento de la carta causando un incremento dramático en la frecuencia de falsas señales. Ante la situación de no detección rápida de pequeños cambios en $\boldsymbol{\theta}$, se requieren estadísticos de control que de alguna forma acumulen información a través de muestras pasadas y en el caso de presencia de no normalidad y/o autocorrelación en los datos es necesario el diseño de cartas de control que sean potentes ante esta situación.

Para detectar pequeños cambios en $\boldsymbol{\theta}$, cartas de control fueron diseñadas con estadísticas de control que acumulen información, entre las que se cuenta la carta EWMA (Roberts 1959) y la CUSUM (Page 1954). Otras cartas alternativas son las cartas con intervalos de muestreo variable, teniendo en cuenta muestreos más eficientes (Reynolds et al. 1990); procedimientos bayesianos y uso de modelos económicos, también han sido considerados (Girshick \& Rubin 1952). Para el caso de no normalidad de las observaciones, se usan procedimientos no paramétricos (Stoumbos \& Jones 2000).

Ahora, para el caso de la autocorrelación que está presente en muchas aplicaciones, particularmente en casos en los cuales los datos están cercanamente espaciados en el tiempo, relativamente, niveles bajos de autocorrelación pueden tener un impacto significativo sobre las propiedades estadísticas de las cartas de control tradicionales diseñadas bajo la condición de independencia. Por ejemplo, la estimación de $\boldsymbol{\theta}_{0}$ puede ser severamente sesgada, resultando una proporción alta de falsas señales de lo que se esperaba bajo el estudio del ARL (Longitud Promedio de Corrida) de la carta, siendo el ARL el número promedio de muestras requeridas para que la carta arroje una señal en el cambio de la media.

Para el monitoreo de observaciones autocorrelacionadas, varias cartas de control han sido desarrolladas para detectar cambios en el proceso en media. Se tienen dos formas de abordar esta temática desde el estudio de las cartas de control estadísticas. Una de ellas se llama cartas de control especiales SCC, basadas sobre los residuales del modelo que mejor se ajuste a los datos trabajados por Alwan \& Roberts (1988), Wardell et al. (1992), y la otra se llama cartas EWMAST aplicada a la estadística EWMA directamente a los datos autocorrelacionados sin identificar los parámetros del proceso trabajados por Montgomery \& Mastrangelo (1991), Schmid (1997), Zhang (1998), Adams \& Tseng (1998) y Lu \& Reynolds (1999a, 1999b). Alwan \& Roberts (1988), Faltin et al. (1997) y Lu \& Reynolds $(1999 b)$ ajustan un modelo de series de tiempo a las observaciones y aplican cartas de control tradicionales al listado de residuales de este modelo, detectando una señal cuando el residual esté fuera de los límites de control. 
Según Montgomery \& Mastrangelo (1991), si las observaciones están autocorrelacionadas en forma positiva y se ajustan a un miembro de la familia ARIMA, la estadística EWMA con un valor apropiado de $\lambda$ (constante entre 0 y 1 ) provee un excelente predictor un paso adelante, además el ajuste se puede realizar a un modelo IMA(1,1), creando con este una carta de control modificada que posee un buen comportamiento ante datos autocorrelacionados. Surge un problema, si un modelo de serie de tiempo realmente captura el comportamiento bajo control del proceso, entonces los parámetros de este modelo se convierten en elementos de $\boldsymbol{\theta}$. Así la complejidad del problema del proceso de monitoreo es incrementada debido al aumento en el número de parámetros a estimar y monitorear. La ventaja de este procedimiento es que se puede trabajar con los datos originales.

Basseville \& Nikiforov (1993) dan una revisión de los algoritmos en el caso univariado de extensiones de la carta CUSUM, de cartas basadas sobre la razón de verosimilitud generalizadas respecto a la distribución de probabilidad de $\boldsymbol{\theta}$ y esquemas de cartas de control de no verosimilitud para datos autocorrelacionados.

Jiang et al. (2000), proponen una nueva carta de control denominada carta ARMA, basada sobre el monitoreo de la estadística ARMA de las observaciones originales. Las cartas SCC dada por Alwan \& Roberts (1988) y EWMAST dada por Zhang (1998), son casos especiales de la carta ARMA. Estudios de simulación muestran que dicha carta es competitiva respecto a las otras cartas mencionadas.

Sheu \& Lu (2008), presentan una carta que es extensión de la carta EWMA, llamada GWMA, la cual sirve para monitorear tanto media como varianza en forma simultánea para datos autocorrelacionados.

\subsection{Modelos $\operatorname{ARMA}(p, q)$ estacionarios}

Cuando los datos presentan estructura de autocorrelación se pueden estructurar mediante modelos de promedios móviles autorregresivos:

$$
\operatorname{ARMA}(p, q):=\varphi_{p}(B) X_{t}=\theta_{q}(B) Z_{t}
$$

donde $\varphi_{p}(B)=1-\varphi_{1} B-\varphi_{2} B^{2}-\ldots-\varphi_{p} B^{p}$ es un polinomio autorregresivo de orden $p, \theta_{q}(B)=1+\theta_{1} B+\theta_{2} B^{2}+\ldots+\theta_{q} B^{q}$ es el polinomio de promedios móviles de orden $q, B$ es el operador de rezago y $Z_{t}$ es una sucesión de "choques" aleatorios independientes e idénticamente distribuidos con media cero y varianza $\sigma^{2}$, conocido como ruido blanco (Box \& Jenkins 1976).

La figura 1 presenta las diferentes etapas que se deben tener en cuenta para el modelamiento de una serie de tiempo propuesto por Box \& Jenkins (1976), el cual se utilizará para la construcción de la carta ARMA para datos autocorrelacionados. 


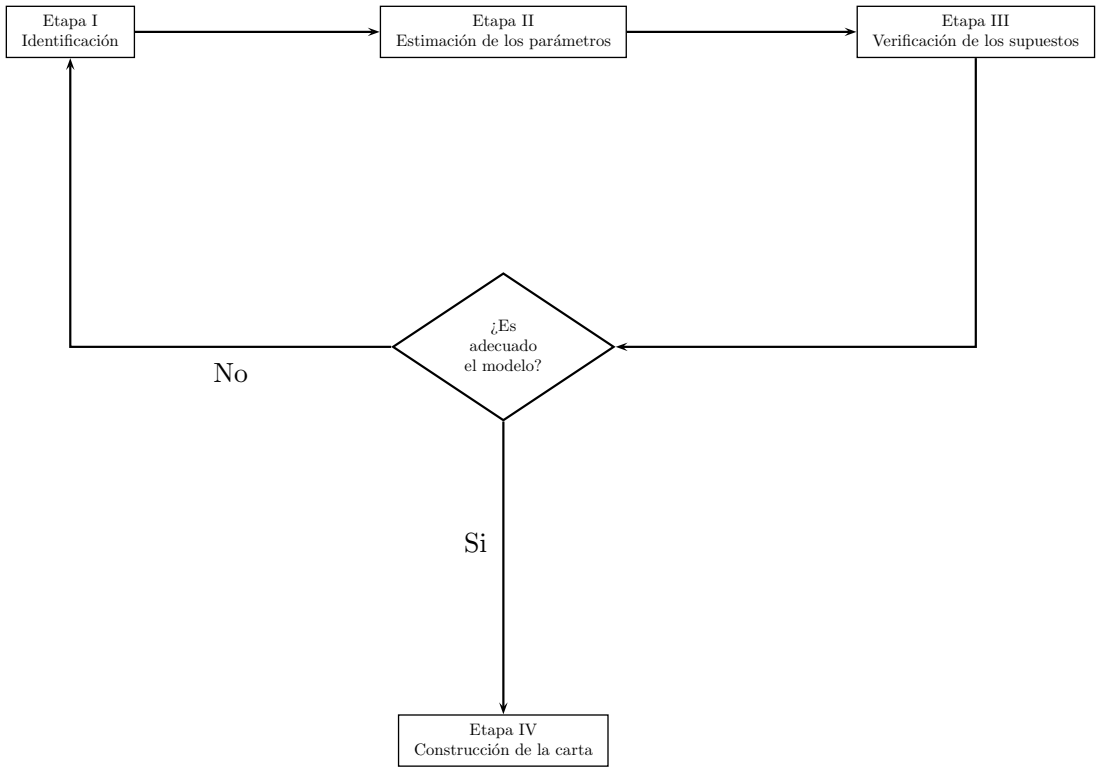

Figura 1: Pasos de la metodología Box-Jenkins para series de tiempo

Para ajustar la serie a un modelo ARMA adecuado en la etapa I (figura11), debemos tener en cuenta que si la serie de tiempo de longitud $n$ presenta tendencia debemos transformarla mediante una diferencia de orden $d$. Posteriormente, en la etapa II, se deben estimar los parámetros del modelo y en la etapa III los residuales son entonces definidos como la diferencia entre un valor observado y su pronóstico un paso adelante, reescalado por la raíz cuadrada del error cuadrático medio (ECM) de la predicción hecha (Brockwell \& Davis 1996); esto es:

$$
\hat{W}_{t}=\frac{X_{t}-\hat{P}_{t-1} X_{t}}{\sqrt{E C M\left(\hat{P}_{t-1} X_{t}\right)}}, \quad t=1,2, \ldots, n,
$$

donde $\hat{P}_{t-1} X_{t}$ es el mejor predictor lineal un paso adelante (bajo mínimo ECM) de $X_{t}$ sobre $\left\{X_{1}, X_{2}, \ldots, X_{t-1}\right\}$. Después de verificar los supuestos en la etapa II se determina que el modelo ajustado es el apropiado, por lo cual $\left\{\hat{W}_{t}\right\}$ debe comportarse aproximadamente como un proceso ruido blanco. El modelo ajustado puede usarse para la construcción de la carta.

\subsection{Carta EWMA para datos autocorrelacionados}

La carta EWMA también puede ser usada en situaciones donde las observaciones están autocorrelacionadas. Supóngase que las observaciones del proceso pueden ser 
modeladas por un $\operatorname{ARIMA}(0,1,1)$, cuyo modelo es

$$
X_{t}=X_{t-1}+\epsilon_{t}-\theta \epsilon_{t-1} .
$$

La estadística EWMA dada por $Z_{t}=\lambda X_{t}+(1-\lambda) Z_{t-1}$ con $\lambda=1-\theta$ es el pronóstico un paso adelante óptimo para este proceso. Es decir, si $\hat{X}_{t+1}(t)$ es el pronóstico para la observación en el periodo $t+1$ hecha al final del periodo $t$, entonces:

$$
\hat{X}_{t+1}(t)=Z_{t} .
$$

La secuencia de errores de predicción un paso adelante es:

$$
e_{t}=X_{t}-\hat{X}_{t}(t-1) \text {. }
$$

Que se comporta aproximadamente como un ruido blanco. Ahora, si el proceso no es exactamente un $\operatorname{ARIMA}(0,1,1)$ pero en lugar es modelado por un miembro de la familia ARIMA y las observaciones del proceso en media no tienen cambios tan rápidamente, el EWMA con un apropiado valor de $\lambda$ (el $\lambda$ que minimice la suma de cuadrados de los errores de predicción un paso adelante $\mathrm{SCE}_{\lambda}$ ) provee un excelente predictor un paso adelante (Montgomery \& Mastrangelo 1991). Los límites de control de la carta sobre los errores satisfacen la siguiente condición de probabilidad

$$
\operatorname{Pr}\left\{-U_{\alpha / 2} \sigma_{p} \leq X_{t}-\hat{X}_{t}(t-1) \leq U_{\alpha / 2} \sigma_{p}\right\},
$$

donde $U_{\alpha / 2}$ es el cuantil $\alpha / 2$ superior de la distribución normal estándar. Pero

$$
\operatorname{Pr}\left\{\hat{X}_{t}(t-1)-U_{\alpha / 2} \sigma_{p} \leq X_{t} \leq \hat{X}_{t}(t-1)+U_{\alpha / 2} \sigma_{p}\right\}=(1-\alpha),
$$

lo que sugiere que el EWMA es un conveniente predictor un paso adelante entonces se puede usar $Z_{t}$ como la línea central sobre una carta de control para el período $t+1$ con:

$$
L C S_{t+1}=Z_{t}+U_{\alpha / 2} \sigma_{p} \quad \text { y } \quad L C I_{t+1}=Z_{t}-U_{\alpha / 2} \sigma_{p} .
$$

Y las observaciones $X_{t+1}$ podrían ser comparadas con estos límites, determinando si el proceso está o no bajo control. Una estimación de $\sigma_{p}$ sugerida por Montgomery (2004) es:

$$
\hat{\sigma}_{p}=\sqrt{\frac{S C E_{\lambda}}{n}} .
$$

\subsection{Carta de residuales para datos autocorrelacionados}

La carta dada por Alwan \& Roberts (1988) que se propone en este artículo también puede ser usada en situaciones donde las observaciones están autocorrelacionadas. Supóngase que las observaciones del proceso pueden ser modeladas por un $\operatorname{ARMA}(p, q)$, cuyo modelo está dado en (11). 
Siguiendo las etapas descritas en la figura 1 se llega al mejor ajuste de los datos, obteniendo las estimaciones de $X_{t}$ denotadas por $\hat{X}_{t}$.

También se obtienen los residuales del modelo dados por

$$
e_{t}=X_{t}-\hat{X}_{t}
$$

Los límites de control de la carta de residuales sobre los errores satisfacen la siguiente condición de probabilidad

$$
\operatorname{Pr}\left\{-U_{\alpha / 2} \sigma_{p} \leq X_{t}-\hat{X}_{t} \leq U_{\alpha / 2} \sigma_{p}\right\},
$$

donde $U_{\alpha / 2}$ es el cuantil $\alpha / 2$ superior de la distribución normal estándar. Pero

$$
\operatorname{Pr}\left\{\hat{X}_{t}-U_{\alpha / 2} \sigma_{p} \leq X_{t} \leq \hat{X}_{t}+U_{\alpha / 2} \sigma_{p}\right\}=(1-\alpha),
$$

Con esto se obtienen los siguientes límites de control

$$
L C S_{t}=\hat{X}_{t}+U_{\alpha / 2} \sigma_{p} \quad \text { y } \quad L C I_{t}=\hat{X}_{t}-U_{\alpha / 2} \sigma_{p} .
$$

Y las observaciones $X_{t}$ podrían ser comparadas con estos límites, determinando si el proceso está o no bajo control. La estimación de $\sigma_{p}$, es:

$$
\hat{\sigma}_{p}=\sqrt{\frac{\sum e_{t}^{2}}{n}}
$$

\section{Comparación ilustrativa de las dos cartas}

A continuación se comparan las cartas EWMA y residuales para datos autocorrelacionados cuya forma de construcción se da en las secciones 2.3 y 2.4 respectivamente.

En un primer ejemplo se considera un conjunto de $n=100$ datos que se simularon mediante el comando arima.sim de la librería tseries (Trapletti \& Hornik 2009) del programa R (R Development Core Team 2009), el cual se ajusta a un modelo de serie tiempo (Figura 2).

De acuerdo a la función de autocorrelación (Figura 3a) y autocorrelación parcial (Figura 3b) se determina que un buen ajuste a los datos es un proceso autorregresivo de orden 1 para una serie estacionaria.

En la estimación de los parámetros del modelo (Tabla 1), se observa que los datos se ajustan a un modelo $\mathrm{AR}(1)$ dado por $\hat{X}_{t}=-0.6592 X_{t-1}$, lo cual nos lleva a determinar que la estructura de autocorrelación de los datos es negativa. 


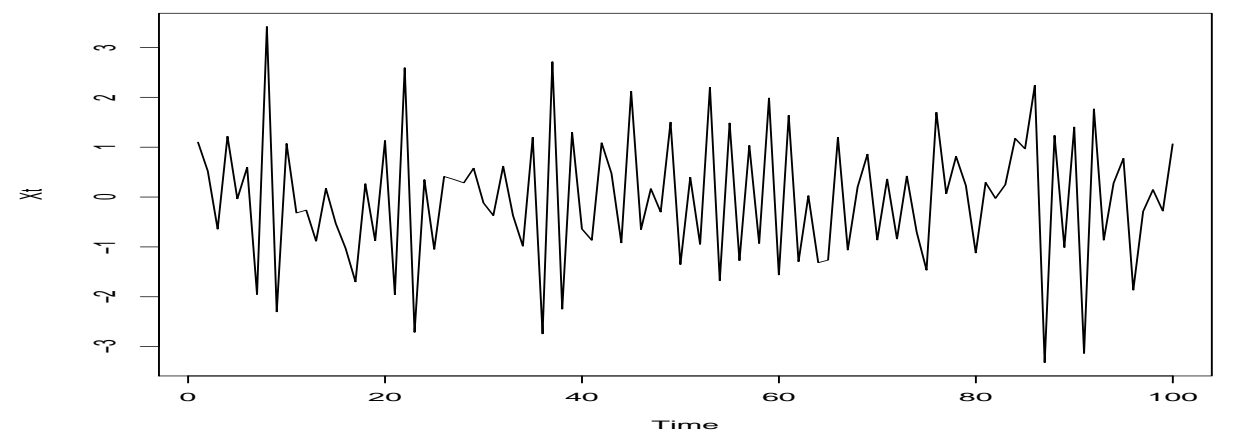

Figura 2: Serie de tiempo con autocorrelación negativa

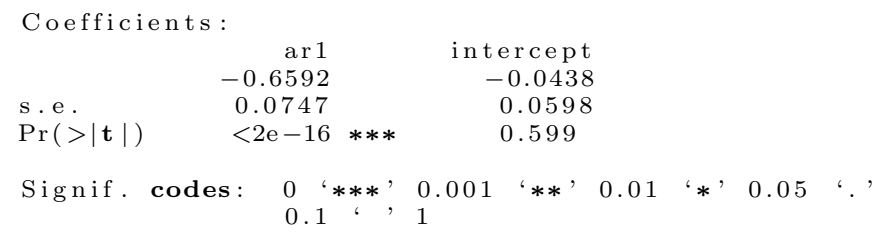

Tabla 1: Estimación de parámetros para la serie figura 2

Se hace el cálculo de los residuales para realizar la verificación de los supuestos del modelo. Según las funciones de autocorrelación y autocorrelación parcial de los residuales (Figura 4), estos tienen un comportamiento de ruido blanco, corroborado con las pruebas analíticas de Jarque y Bera para el caso de la normalidad y la prueba Box-Pierce para independencia de los residuales (Tabla 2).

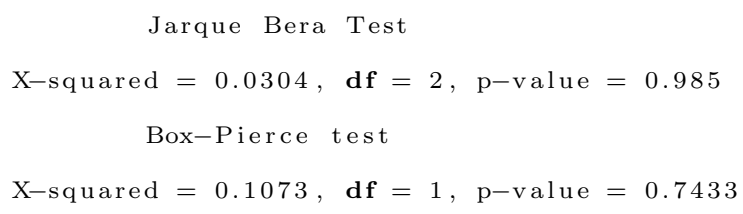

Tabla 2: Pruebas de normalidad e independencia de los residuales del modelo ajustado a los datos 


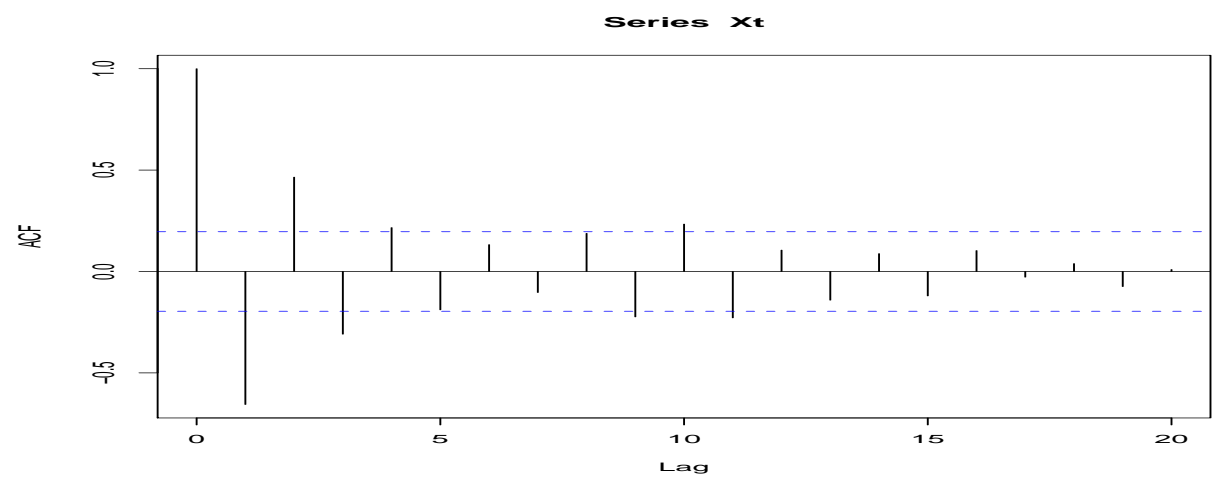

(a) Función de autocorrelación

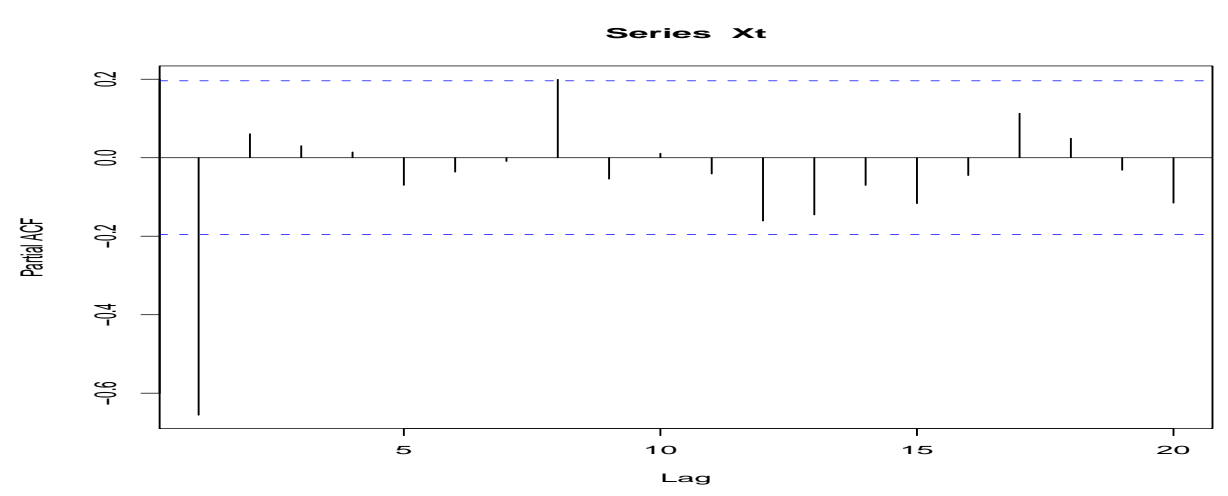

(b) Función de autocorrelación parcial

Figura 3: Función de autocorrelación y de autocorrelación parcial

Observando la gráfica CUSUM (Figura 5a) los datos poseen un buen ajuste al modelo considerado, mientras que el gráfico CUSUMSQ (Figura 5b) nos indica que existe homogeneidad de varianza en los residuales. Luego, se concluye que el modelo al cual se ajustaron los datos es el apropiado.

Realizando el cálculo de la suma de cuadrados de los residuales descrito en la sección 2.3. para valores $0<\lambda \leq 1$, se determina que el valor de $\lambda$ que minimiza la suma de cuadrados de los residuales es $\lambda=0.001$ y con esto se estima $\sigma_{p}$ con la ecuación (22), nos da $\hat{\sigma}_{p}=1.316614$. 


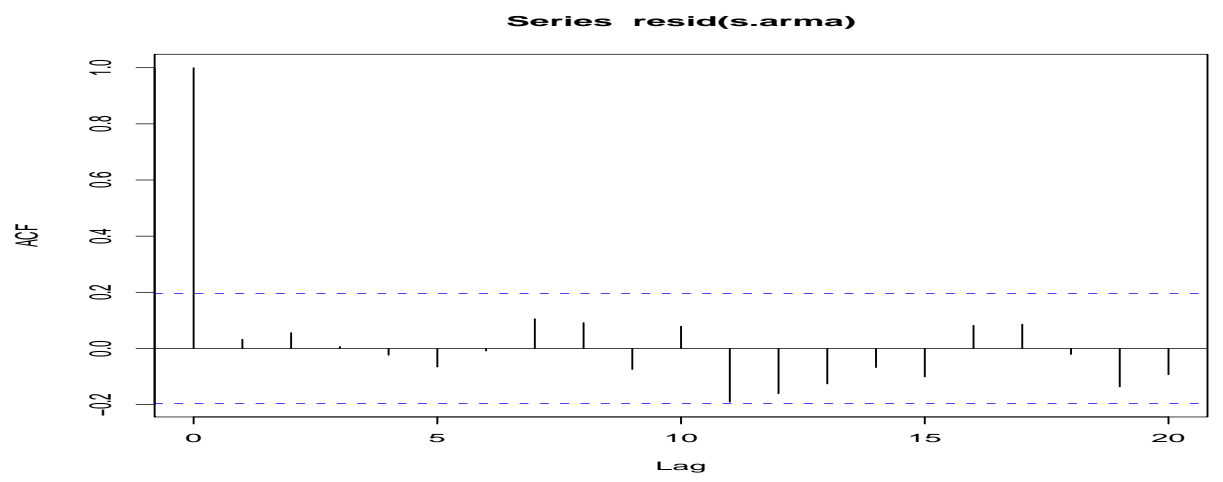

(a) Función de autocorrelación

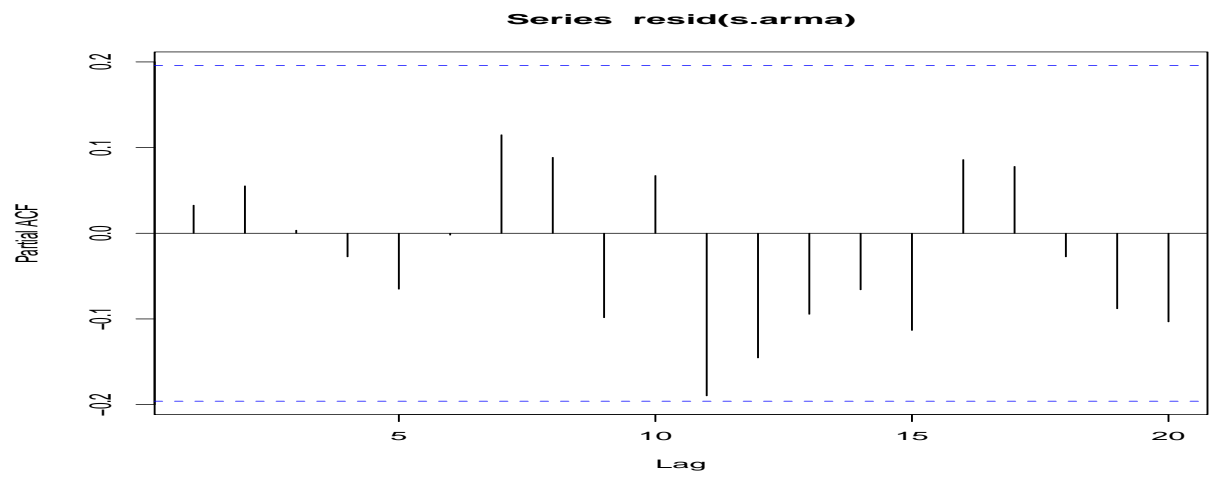

(b) Función de autocorrelación parcial

Figura 4: Funciones de autocorrelación y autocorrelación parcial de los residuales

$\mathrm{Al}$ tener en cuenta la construcción de las dos cartas descritas en las secciones 2.3 y 2.4 se observa que no se tiene un comportamiento similar, debido a que la carta propuesta por Montgomery \& Mastrangelo (1991) (Figura 6a) se distorsiona, mientras que en la carta de residuales propuesta por Alwan \& Roberts (1988) (Figura 6b), su construcción posee límites de control móviles siendo la característica de este tipo de cartas.

Haciendo el mismo procedimiento anterior, se simula un conjunto de datos con autocorrelación positiva que se ajusta a un modelo $\operatorname{AR}(1)$ con $\hat{\phi}=0.8$, obteniéndose las cartas de la figura 7. En este caso se observa un comportamiento similar en los dos tipos de cartas consideradas.

Para la construcción de las gráficas de sumas de cuadrados de los residuales y la carta de control propuesta por Montgomery \& Mastrangelo (1991), los códigos en R se pueden consultar en J. \& J. (2008) y se describen en el apéndice de este artículo. 


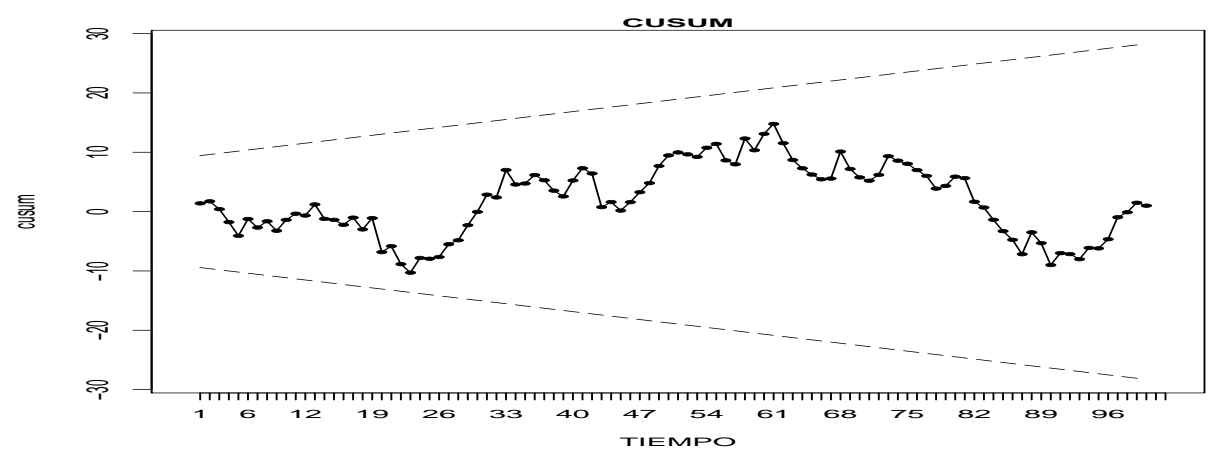

(a) Gráfico CUSUM

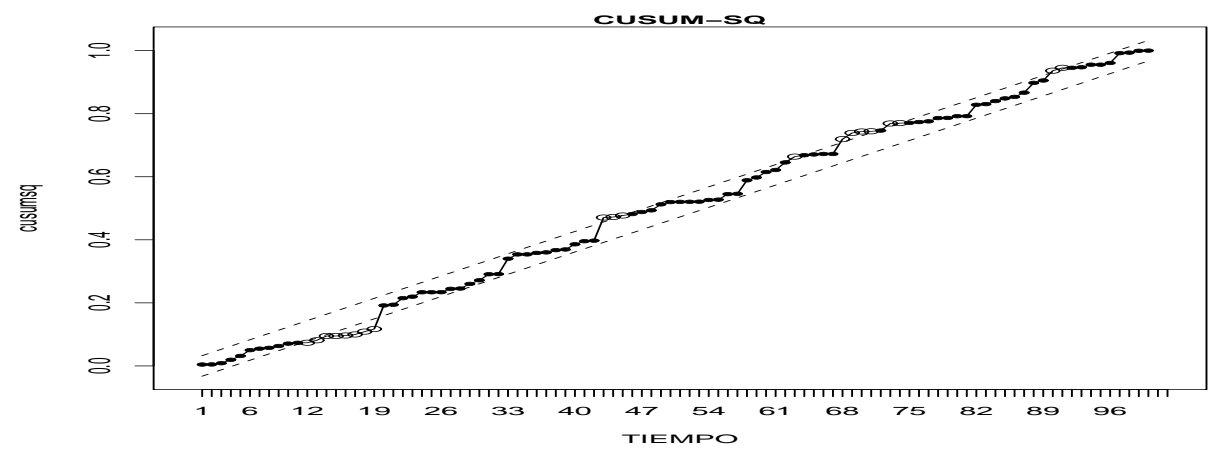

(b) Gráfico CUSUMSQ

Figura 5: Gráficos CUSUM y CUSUMSQ de los residuales

\section{Conclusiones}

En este trabajo se presentó la construcción y comparación de dos propuestas de cartas de control que están diseñadas para trabajar con datos autocorrelacionados. Se realizó su implementación mediante el uso de comandos y programación del paquete estadístico de uso libre R. En la construcción de las cartas se tiene en cuenta la estructura del ajuste de los datos a un modelo en series de tiempo tipo ARMA y de la estadística EWMA. Se consideran los casos cuando la autocorrelación de los datos es positiva y cuando es negativa llegando a las siguientes conclusiones:

1. La carta propuesta dada por Alwan \& Roberts (1988) no posee inconvenientes ante autocorrelaciones negativas, contrario a lo que sucede con la carta propuesta por Montgomery \& Mastrangelo (1991),

2. Un inconveniente que puede presentar la construcción de la carta propuesta por Alwan \& Roberts (1988) es la identificación del modelo tipo ARMA que 


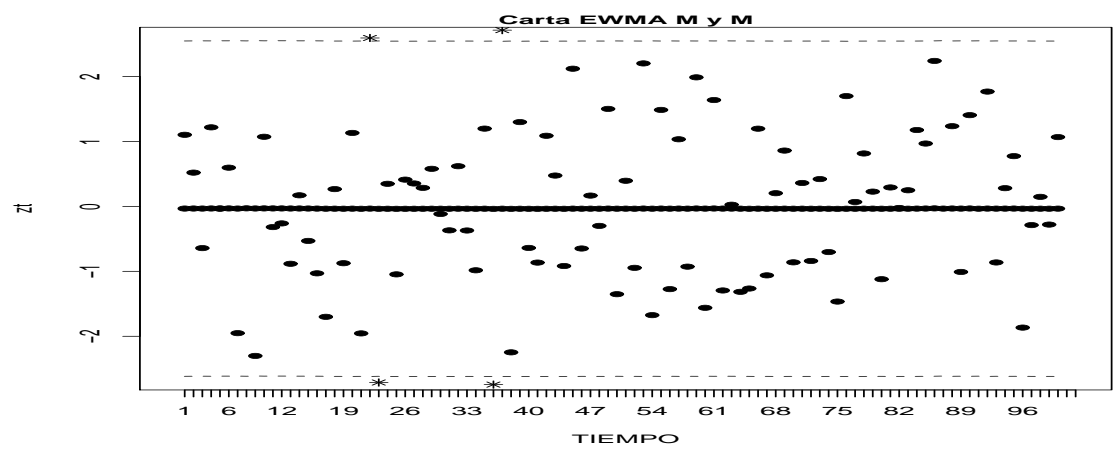

(a) Carta propuesta por Montgomery y Mastrangelo (1991)

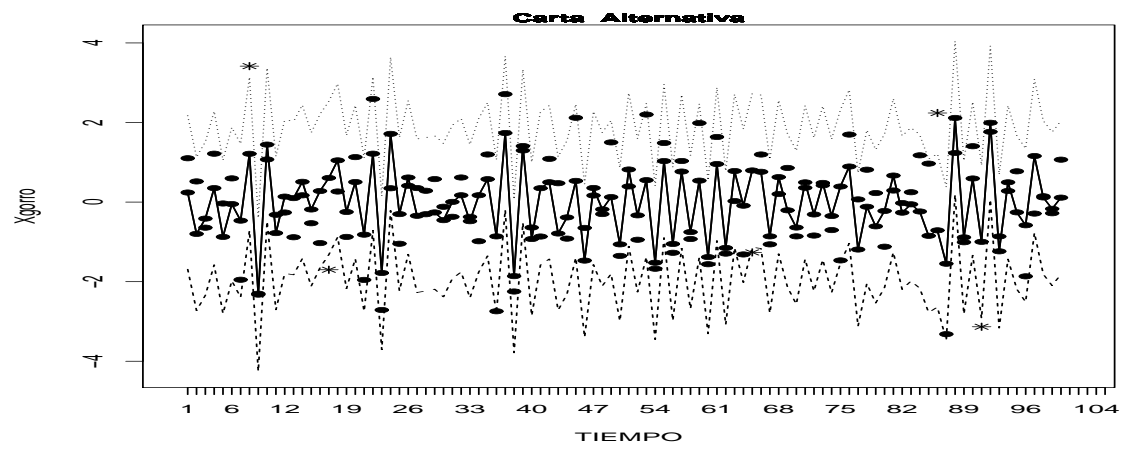

(b) Carta ARMA

Figura 6: Cartas de control para datos autocorrelacionados en forma negativa

mejor se ajuste a los datos, problema no presentado en la carta propuesta por Montgomery \& Mastrangelo (1991), ya que esta sólo considera un ajuste a un IMA $(1,1)$ por la naturaleza de la estadística EWMA siempre y cuando la autocorrelación de los datos sea positiva y el cambio en media no sea tan drástico.

Las anteriores conclusiones son dadas en los respectivos artículos donde se proponen las cartas, aquí se llega mediante el análisis de los ejemplos simulados dados. Por tal razón, la implementación de estas cartas se hizo con el propósito de poseer una herramienta didáctica en la enseñanza y aprendizaje de cartas de control especiales en un curso de control de calidad a nivel de pregrado. La implementación y análisis de otro tipo de cartas especiales no incluidas en las librerías especializadas de paquetes estadísticos es una buena alternativa de estudio y de investigación a nivel de programas de pregrado de matemáticas y estadística. 


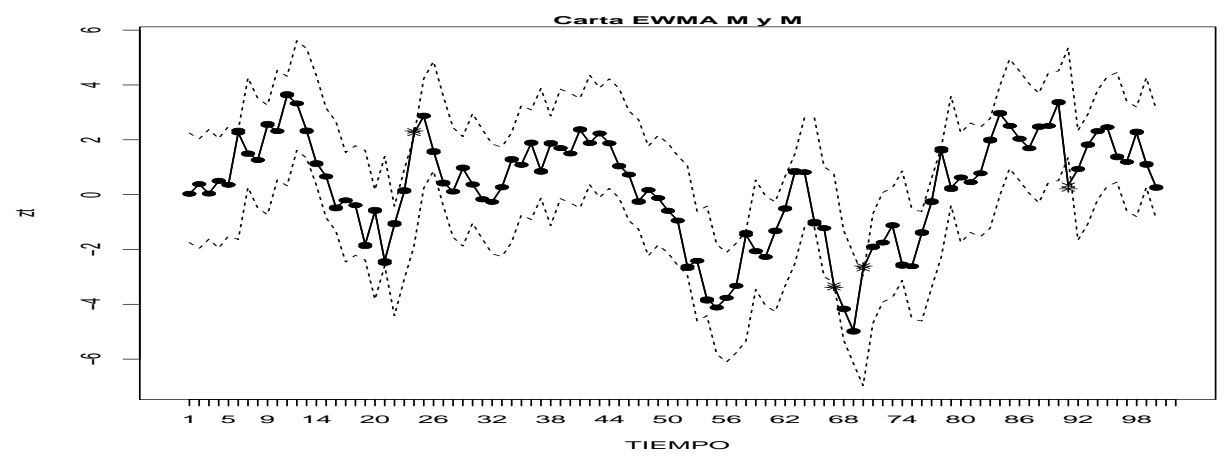

(a) Carta propuesta por Montgomery y Mastrangelo (1991)

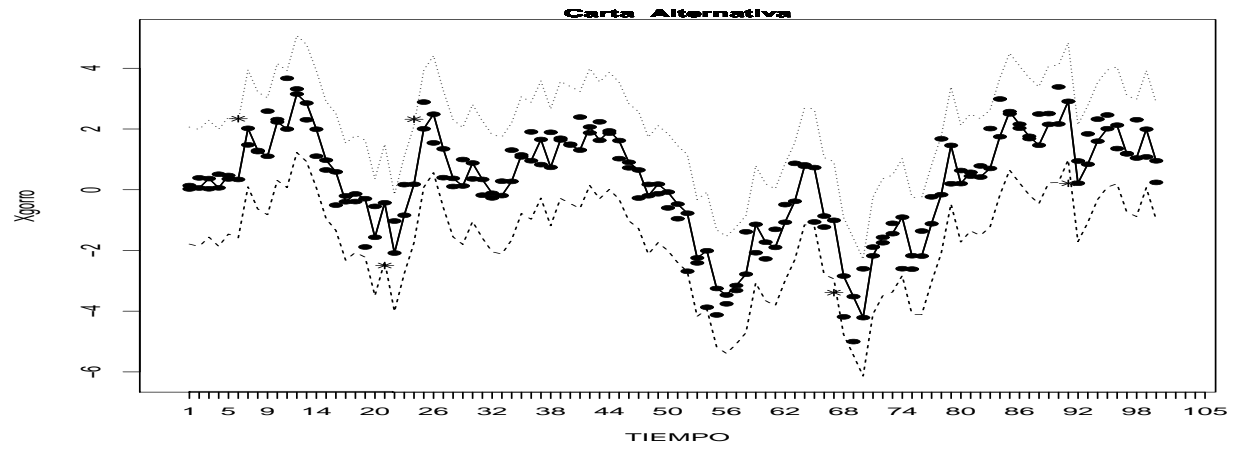

(b) Carta ARMA

Figura 7: Cartas de control para datos autocorrelacionados en forma positiva

Recibido: 30 de noviembre de 2010 Aceptado: 1 de marzo de 2011

\section{Referencias}

Adams, B. M. \& Tseng, I.-T. (1998), 'Robustness of Forecast-Based Monitoring Schemes', Journal of Quality Technology 30, 328-339.

Alwan, L. C. \& Roberts, H. V. (1988), 'Time-Series Modeling for Statistical Process Control', Journal of Business and Economic Statistics 6(1), 87-95.

Basseville, M. \& Nikiforov, I. V. (1993), Detection of Abrupt Changes: Theory and Applications, Prentice-Hall, Engelwoold Cliffs, NJ. 
Box, G. E. P. \& Jenkins, G. M. (1976), Time Series Analysis, Forecasting, and Control, Holden Day, Oakland, C.A.

Brockwell, P. J. \& Davis, R. A. (1996), Introduction to Time Series and Forecasting, Springer-Verlag, New York.

Faltin, F. W., Mastrangelo, C. M., Runger, G. C. \& Ryan, T. P. (1997), 'Considerations in the Monitoring of Autocorrelated and Independent Data', Journal of Quality Technology 29(2), 131-133.

Girshick, M. A. \& Rubin, H. (1952), 'A Bayes Approach to a Quality Control Model', The Annals of Mathematical Statistics 23, 114-125.

J., G. B. \& J., P. P. (2008), 'Estudio de la carta EWMA en presencia de datos autocorrelacionados', Entre Ciencia e Ingeniería (3), 11-25.

Jiang, W., Tsui, K.-L. \& Woodall, W. H. (2000), 'A New SPC Monitoring Method: The ARMA Chart', Technometrics 42(4), 399-410.

Lu, C. W. \& Reynolds, Jr., M. R. (1999a), 'Control Charts for Monitoring the Mean of Autocorrelated the Mean and Variance Processes', Journal of Quality Technology 31(3), 259-274.

Lu, C. W. \& Reynolds, Jr., M. R. (1999b), 'EWMA Control Charts for Monitoring the Mean of Autocorrelated Processes', Journal of Quality Technology 31(2), 166-188.

Montgomery, D. C. (2004), Control Estadístico de la Calidad, 3a. edn, Limusa Wiley, México.

Montgomery, D. C. \& Mastrangelo, C. M. (1991), 'Some Statistical Process Control Methods for Autocorrelated Data', Journal of Quality Technology 23(3), 179204.

Page, E. S. (1954), 'Continuous Inspection Schemes', Biometrika 41, 100-114.

R Development Core Team (2009), R: A Language and Environment for Statistical Computing, R Foundation for Statistical Computing, Vienna, Austria.

Reynolds, M. R., Amin, R. W. \& Arnold, J. C. (1990), 'CUSUM Charts With Variable Sampling Intervals', Technometrics 32, 371-384.

Roberts, S. W. (1959), 'Control Chart Tests Based on Geometrics Moving Averages', Technometrics 1, 239-250.

Schmid, W. (1997), On EWMA Charts for Time Series, Vol. 5, Frontiers in Statistical Quality Control, Physica-Verlag.

Sheu, S.-H. \& Lu, S.-L. (2008), 'Monitoring Autocorrelated Process Mean and Variance using a GWMA Chart based on Residuals', Asia - Pacific Journal of Operational Research 25(6), 781-792. 
Stoumbos, Z. G. \& Jones, L. A. (2000), 'On the Properties and Design of Individuals Control Charts Based on Simplicial Depth', Nonlinear Studies 7, 147178 .

Trapletti, A. \& Hornik, K. (2009), Tseries: Time Series Analysis and Computational Finance. R package version 0.10-22.

*http://CRAN.R-project.org/package=tseries

Wardell, D. G., Moskowitz, H. \& Plante, R. D. (1992), 'Control Charts in the Presence of Data Correlation', Management Science 38, 1084-1105.

Zhang, N. F. (1998), 'A Statistical Control Chart for Stationary Process Data', Technometrics 40, 24-38.

\section{Apéndice}

En este apéndice se presentan los algoritmos para el ajuste de los datos a un modelo ARMA, para la construcción de la carta propuesta por Montgomery \& Mastrangelo (1991) y la carta de residuales propuesta por Alwan \& Roberts (1988).

\section{A. Ajuste de los datos a un modelo ARMA}

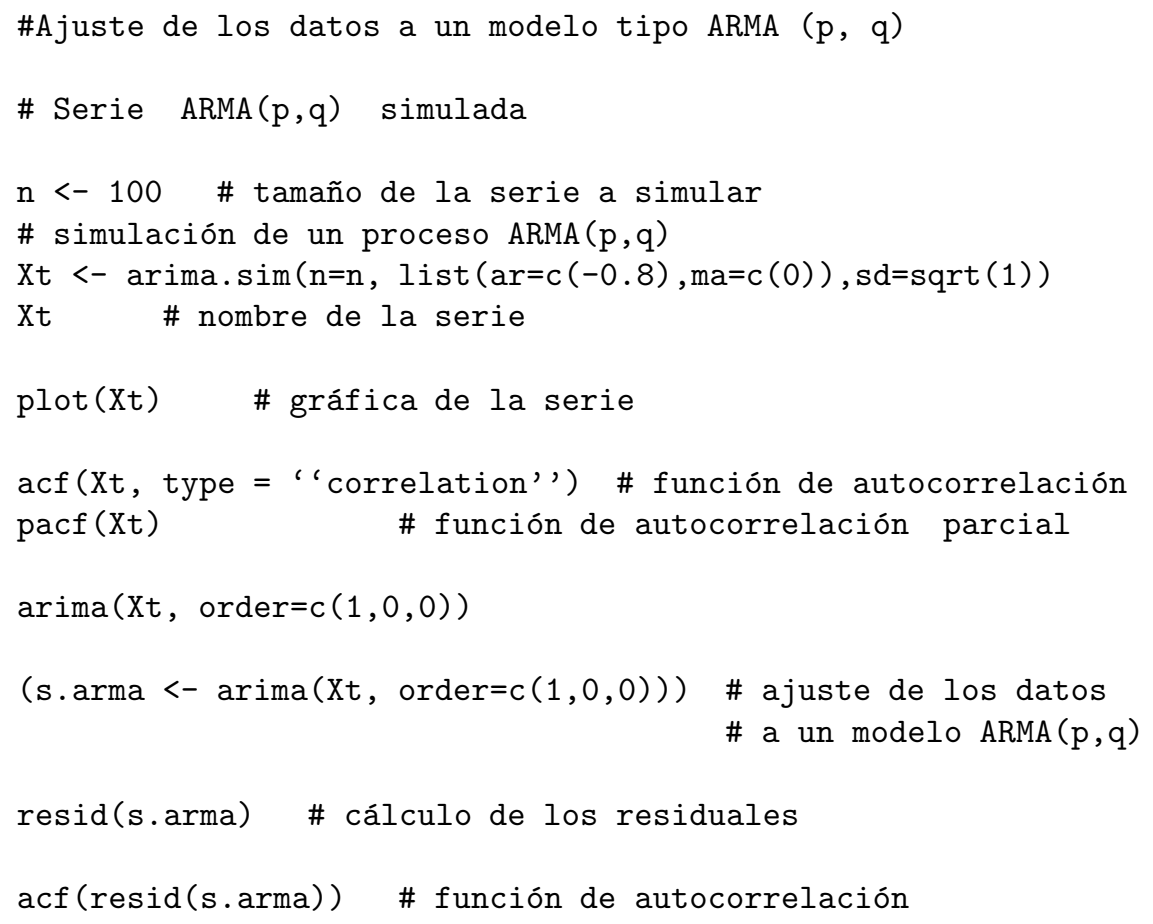




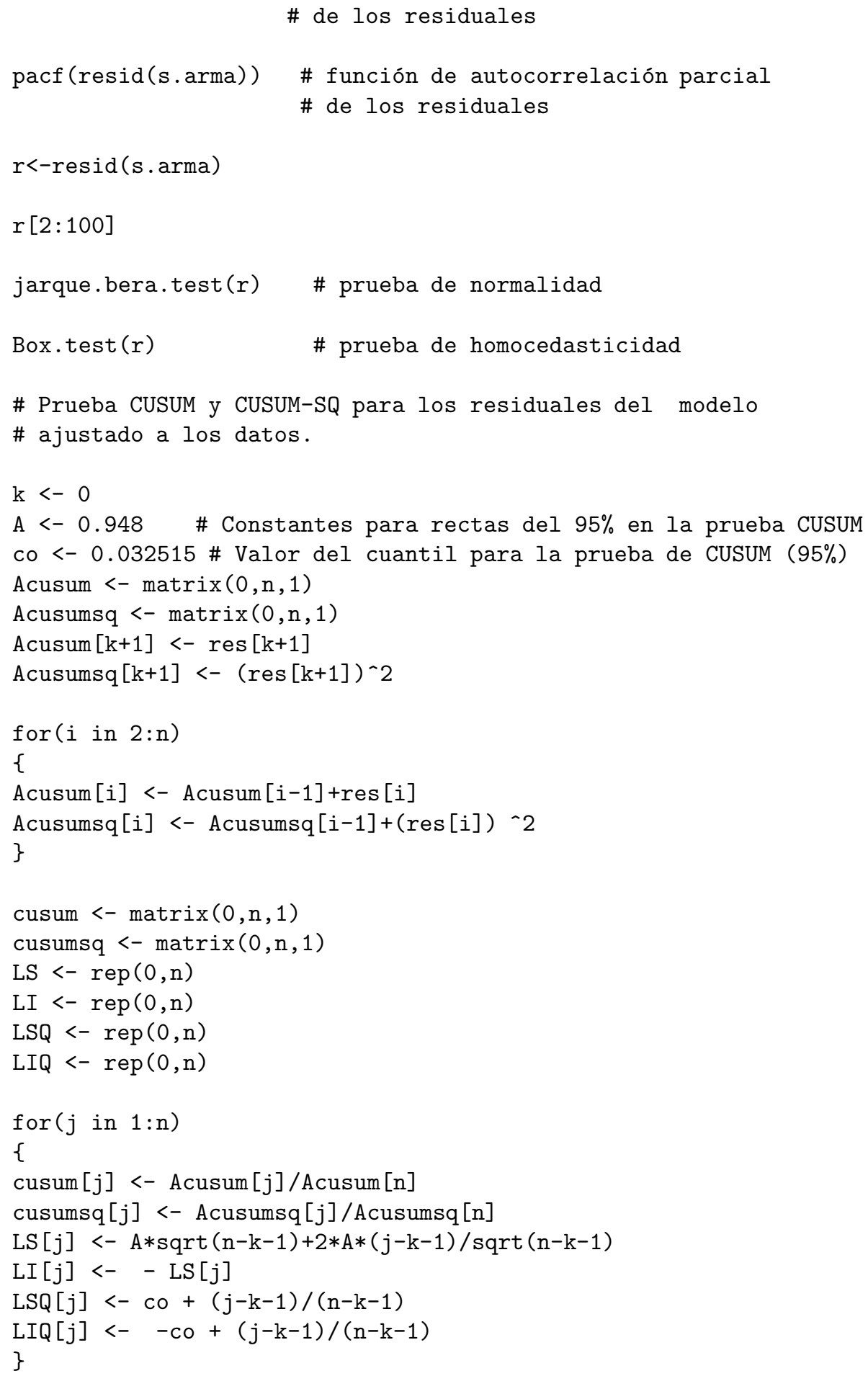




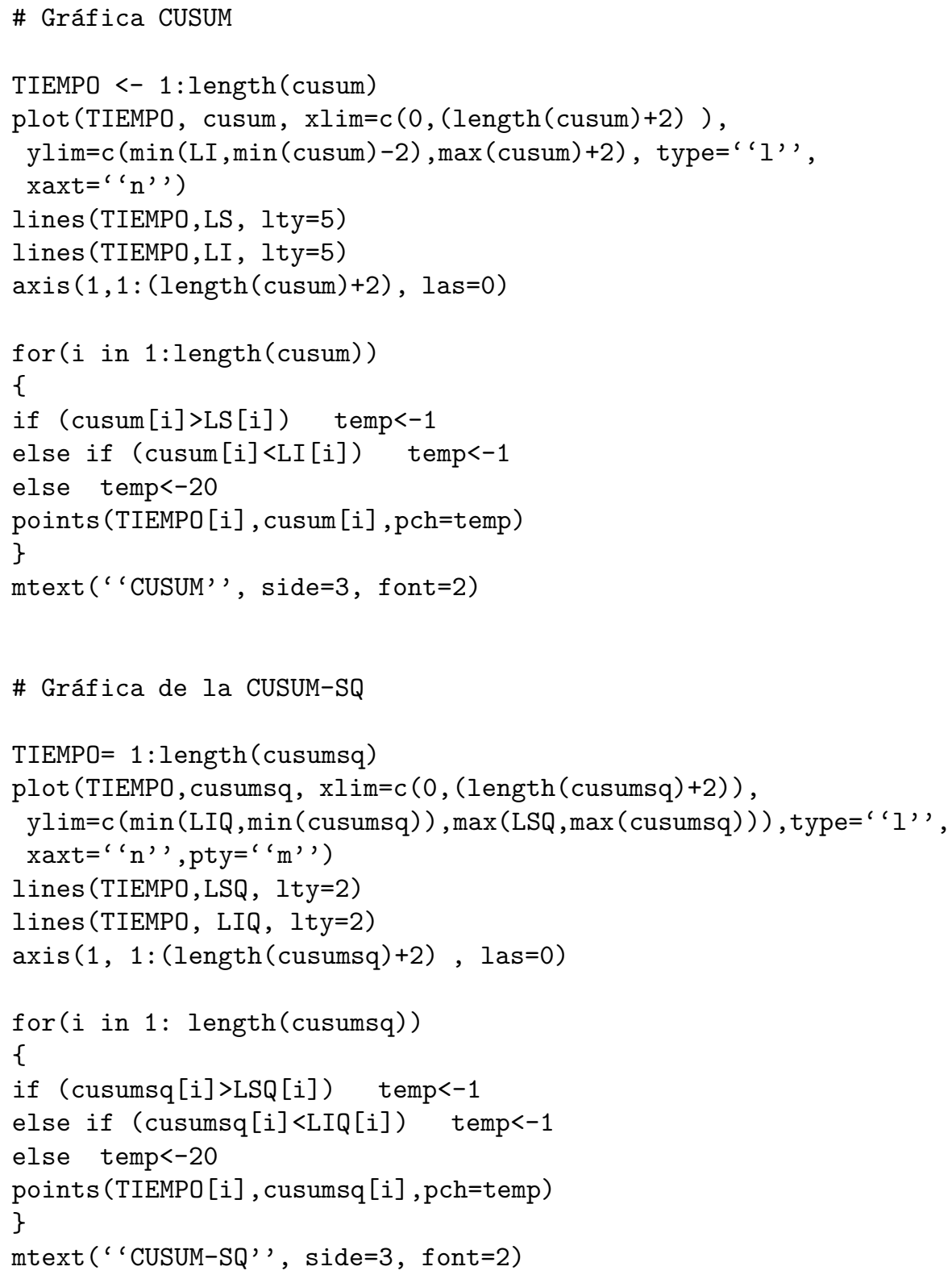

\section{B. Programa para la carta EWMA}

\# Cálculo de la matriz del estadístico EWMA variando el valor de lambda 


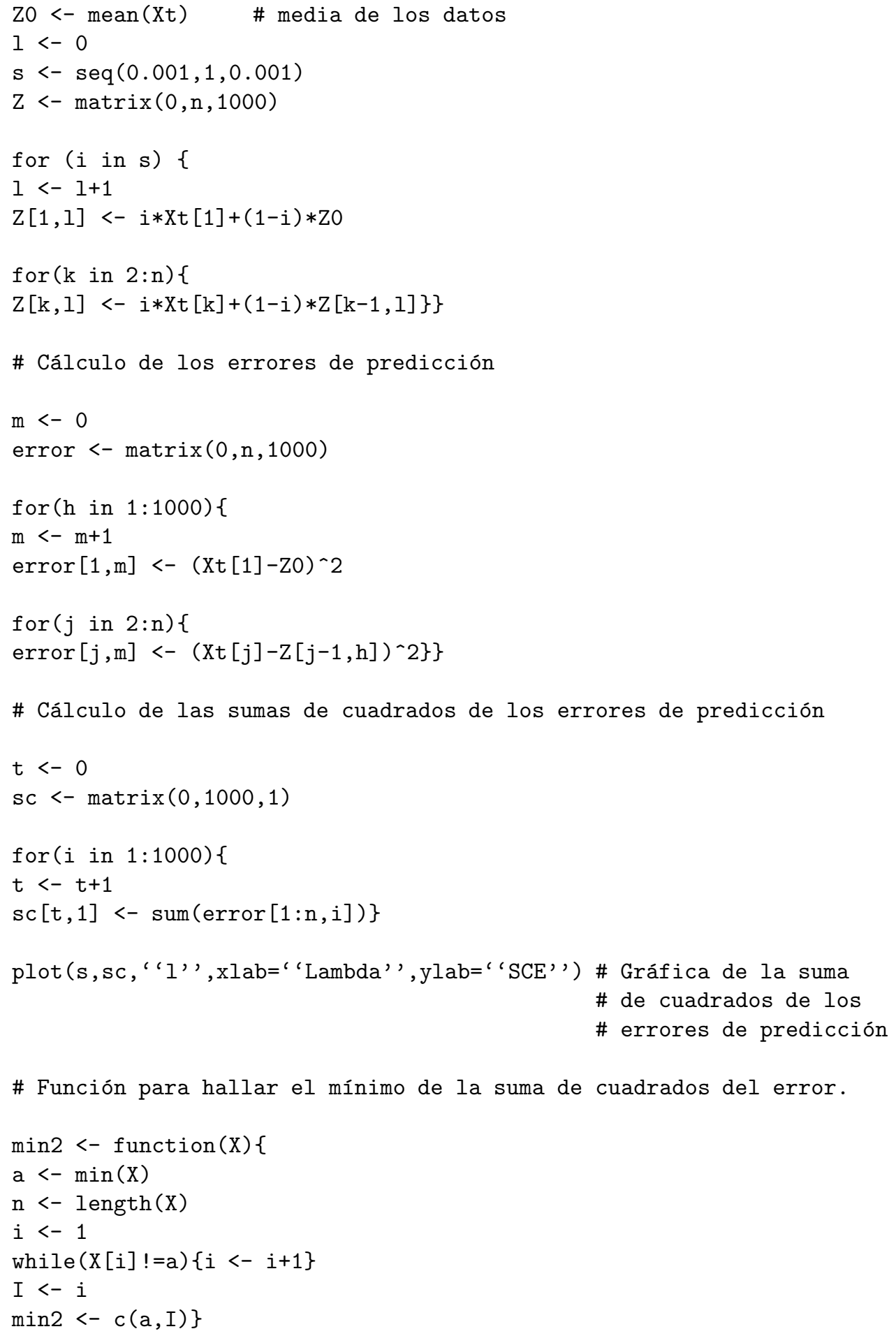




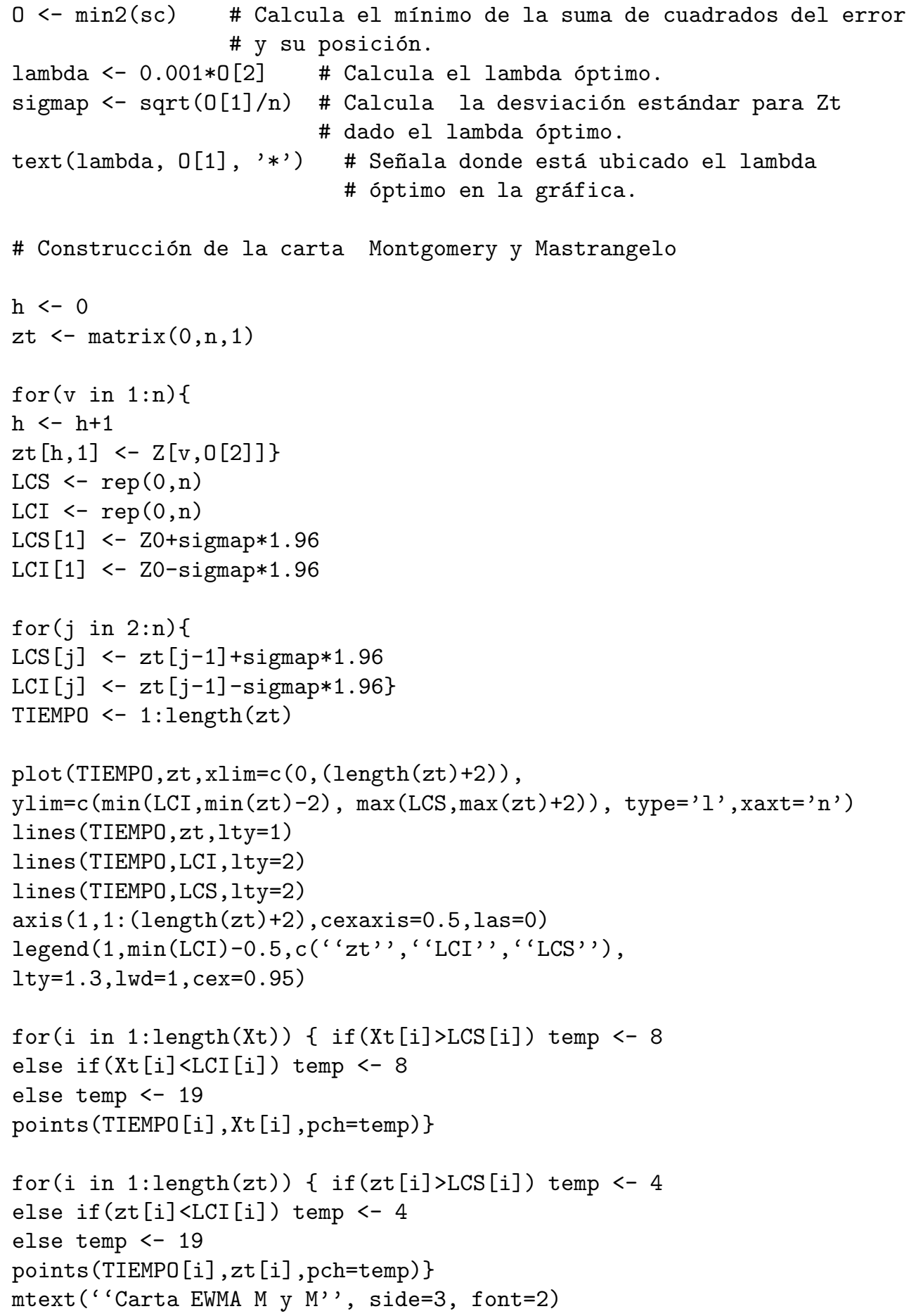




\section{Programa para las cartas residuales}

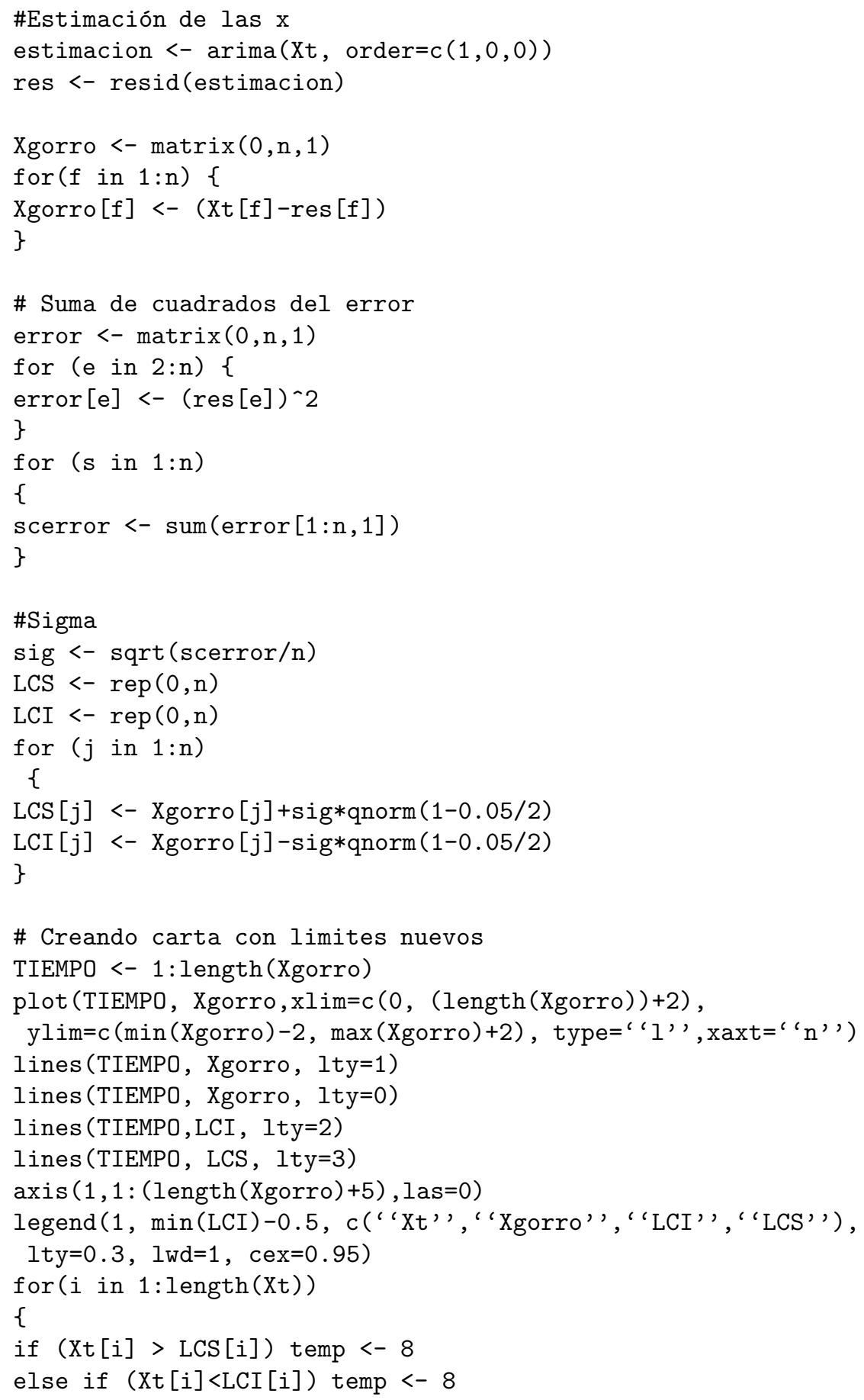




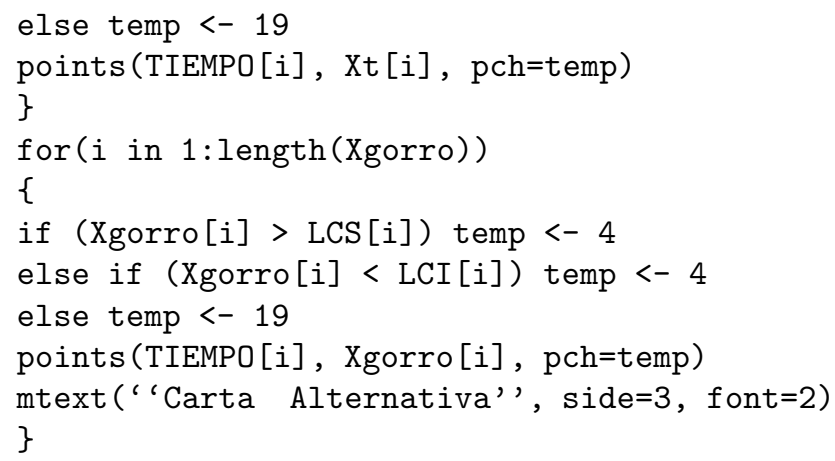

